\title{
NONLINEAR SCHRÖDINGER EQUATION FOR THE TWISTED LAPLACIAN IN THE CRITICAL CASE
}

\author{
VIJAY KUMAR SOHANI
}

\begin{abstract}
In [6] and [7, we prove well-posedness of solution to the nonlinear Schrödinger equation associated to the twisted Laplacian on $\mathbb{C}^{n}$ for a general class of nonlinearities including power type with subcritical case $0 \leq \alpha<\frac{2}{n-1}$. In this paper, we consider critical case $\alpha=\frac{2}{n-1}$ with $n \geq 2$. Our approach is based on truncation of the given nonlinearity $G$, which is used in 3 . We obtain solution for the truncated problem. We obtain solution to the original problem by passing to the limit.
\end{abstract}

\section{InTRODUCTION}

We consider the initial value problem for the nonlinear Schrödinger equation for the twisted Laplacian $\mathcal{L}$ :

$$
\begin{aligned}
i \partial_{t} u(z, t)-\mathcal{L} u(z, t) & =G(z, u), z \in \mathbb{C}^{n}, t \in \mathbb{R} \\
u\left(z, t_{0}\right) & =f(z) .
\end{aligned}
$$

Here we consider the nonlinearity $G$ of the form

$$
G(z, w)=\psi(x, y,|w|) w,(x, y, w) \in \mathbb{R}^{n} \times \mathbb{R}^{n} \times \mathbb{C},
$$

where $z=x+i y \in \mathbb{C}^{n}, w \in \mathbb{C}$ and $\psi \in C\left(\mathbb{R}^{n} \times \mathbb{R}^{n} \times[0, \infty)\right) \cap C^{1}\left(\mathbb{R}^{n} \times \mathbb{R}^{n} \times\right.$ $(0, \infty))$ satisfy the following inequality

$$
|F(x, y, \eta)| \leq C|\eta|^{\alpha}
$$

with $F=\psi, \partial_{x_{j}} \psi, \partial_{y_{j}} \psi(1 \leq j \leq n)$ and $\eta \partial_{\eta} \psi(x, y, \eta), \alpha=\frac{2}{n-1}$ with $n \geq 2$ and for some constant $C$.

In [7], we consider subcritical case $0 \leq \alpha<\frac{2}{n-1}$ for initial value in $\tilde{W}_{\mathcal{L}}^{1,2}\left(\mathbb{C}^{n}\right)$. Sobolev space $\tilde{W}_{\mathcal{L}}^{1, p}\left(\mathbb{C}^{n}\right)$ is introduced in [7]. In this paper, we consider the critical case $\alpha=\frac{2}{n-1}$. In subcritical case $0 \leq \alpha<\frac{2}{n-1}$ for each $\alpha$, we have some $q>2$ such that $(q, 2+\alpha)$ be an admissible pair (see Definition 3.1 in [7]), which is not the case when $\alpha=\frac{2}{n-1}$. We overcome this difficulty by

2010 Mathematics Subject Classification. Primary 42B37, Secondary 35G20, 35G25.

Key words and phrases. Twisted Laplacian(special Hermite operator), Nonlinear Schrödinger equation, Strichartz estimates, well posedness . 
considering admissible pair $(\gamma, \rho)$ and by using embedding theorem (Lemma 2.1), where

$$
\rho=\frac{2 n^{2}}{n^{2}-n+1}, \gamma=\frac{2 n}{n-1}
$$

To treat the critical case, we adopt truncation argument method of Cazenave and Weissler [3]. To prove local existence, we truncate the given nonlinearity $G$ and obtain solution for the truncated problem. Now we obtain solution $u$ for given nonlinearity $G$ by using Strichartz estimates and by passing to the limit.

The twisted Laplacian operator $\mathcal{L}$ was introduced by R. S. Strichartz [8], and called the special Hermite operator. The twisted Laplacian $\mathcal{L}$ on $\mathbb{C}^{n}$ is given by

$$
\mathcal{L}=\frac{1}{2} \sum_{j=1}^{n}\left(Z_{j} \bar{Z}_{j}+\bar{Z}_{j} Z_{j}\right)
$$

where $Z_{j}=\frac{\partial}{\partial z_{j}}+\frac{1}{2} \bar{z}_{j}, \bar{Z}_{j}=-\frac{\partial}{\partial \bar{z}_{j}}+\frac{1}{2} z_{j}, j=1,2, \ldots n$. Here $\frac{\partial}{\partial z_{j}}$ and $\frac{\partial}{\partial \bar{z}_{j}}$ denote the complex derivatives $\frac{\partial}{\partial_{x_{j}}} \mp i \frac{\partial}{\partial_{y_{j}}}$ respectively. Nonlinear Schrödinger equation for the twisted Laplacian has been studied in [6, 7, 12]. For spectral theory of twisted Laplacian $\mathcal{L}$ we refer to [9, 10] and for Schrödinger equation we refer to [2, 11]. Now we state the main theorem of this paper.

Theorem 1.1. Let $f \in \tilde{W}_{\mathcal{L}}^{1,2}\left(\mathbb{C}^{n}\right)$ and $G$ be as in (1.3) and (1.4) with $\alpha=$ $\frac{2}{n-1}$ and $n \geq 2$. Initial value problem (1.1), (1.2) has maximal solution $u \in$ $C\left(\left(T_{*}, T^{*}\right), \tilde{W}_{\mathcal{L}}^{1,2}\right) \cap L_{\text {loc }}^{q_{1}}\left(\left(T_{*}, T^{*}\right), \tilde{W}_{\mathcal{L}}^{1, p_{1}}\left(\mathbb{C}^{n}\right)\right)$ for every admissible pair $\left(q_{1}, p_{1}\right)$, where $t_{0} \in\left(T_{*}, T^{*}\right)$. Moreover the following properties hold:

(i)(Uniqueness): Solution is unique in $C\left(\left(T_{*}, T^{*}\right), \tilde{W}_{\mathcal{L}}^{1,2}\left(\mathbb{C}^{n}\right)\right) \cap L^{\gamma}\left(\left(T_{*}, T^{*}\right)\right.$, $\left.\tilde{W}_{\mathcal{L}}^{1, \rho}\right)$.

(ii)(Blowup alternative): If $T^{*}<\infty$ then $\|u\|_{L^{q}\left(\left(t_{0}, T^{*}\right), \tilde{W}_{\mathcal{L}}^{1, p}\right)}=\infty$ for every admissible pair $(q, p)$ with $2<p$ and $\frac{1}{q}=n\left(\frac{1}{2}-\frac{1}{p}\right)$. Similar conclusion holds if $T_{*}>-\infty$.

(iii) (Stability): If $f_{j} \rightarrow f$ in $\tilde{W}_{\mathcal{L}}^{1,2}\left(\mathbb{C}^{n}\right)$ then $\left\|u-\tilde{u}_{j}\right\|_{L^{q}\left(I, \tilde{W}_{\mathcal{L}}^{1, p}\left(\mathbb{C}^{n}\right)\right)} \rightarrow 0$ as $j \rightarrow \infty$ for every admissible pair $(q, p)$ and every interval $I$ with $\bar{I} \subset$ $\left(T_{*}, T^{*}\right)$, where $u, \tilde{u}_{j}$ are solutions corresponding to $f, f_{j}$ respectively.

(iv)(Conservation of charge and energy): If $\psi: \mathbb{C}^{n} \times[0, \infty) \rightarrow \mathbb{R}$ is real valued, then we have conservation of charge, i.e., $\|u(\cdot, t)\|_{L^{2}\left(\mathbb{C}^{n}\right)}=$ $\|f\|_{L^{2}\left(\mathbb{C}^{n}\right)}$ and conservation of energy $E(u(\cdot, t))=E\left(u\left(\cdot, t_{0}\right)\right)=E(f)$ 
for each $t \in\left(T_{*}, T^{*}\right)$, where

$$
E(f)=\frac{1}{4} \sum_{j=1}^{n}\left(\left\|Z_{j} f\right\|_{L^{2}\left(\mathbb{C}^{n}\right)}^{2}+\left\|\bar{Z}_{j} f\right\|_{L^{2}\left(\mathbb{C}^{n}\right)}^{2}\right)+\int_{\mathbb{C}^{n}} \tilde{G}(z,|f|) d z
$$

and $\tilde{G}: \mathbb{C}^{n} \times[0, \infty) \rightarrow \mathbb{R}$ is given by

$$
\tilde{G}(z, \sigma)=\int_{s=0}^{\sigma} s \psi(z, s) d s=\int_{s=0}^{\sigma} G(z, s) d s .
$$

To prove local existence, we truncate the given nonlinearity $G$ and obtain solutions for the truncated problem. For $m \geq 1$, consider $G_{m}(z, u)=$ $\psi_{m}(z,|u|) u: \mathbb{C}^{n} \times \mathbb{C} \rightarrow \mathbb{C}$, where

$$
\psi_{m}(z, \sigma)=\left\{\begin{array}{cc}
\psi(z, \sigma) & \text { if } 0 \leq \sigma \leq m \\
m^{2}\left(\frac{\psi(z, \sigma)}{\sigma^{2}}-\frac{\psi(z, m)}{\sigma^{2}}+\frac{\psi(z, m)}{m^{2}}\right) & \text { if } \sigma \geq m
\end{array}\right.
$$

For $m=0$, we define $G_{0}(z, u)=G(z, u)$ and $\psi_{0}(z,|u|)=\psi(z,|u|)$. Note that $\psi_{m}$ is differentiable at $\sigma=m$ with respect to $\sigma$ and also note that $G_{m}$ will satisfy (1.3) and (1.4) with $\alpha=\frac{2}{n-1}$ as well as $\alpha=0$. For $m \geq 1$, $G_{m}(z, \cdot): \mathbb{C} \rightarrow \mathbb{C}$ is globally Lipschitz from mean value theorem and

$$
\left|G_{m}(z, u)-G_{m}(z, v)\right| \leq C_{m}|u-v| \text { for } m \geq 1
$$

where constant $C_{m}$ depends on $m \in \mathbb{Z}_{\geq 1}$ but independent of $z \in \mathbb{C}^{n}$ and $u, v \in C$. Moreover by mean value theorem we also see that

$$
\left|G_{m}(z, u)-G_{m}(z, v)\right| \leq C(|u|+|v|)^{\frac{2}{n-1}}|u-v| \text { for } m \geq 0
$$

where constant $C$ is independent of $m \in \mathbb{Z}_{\geq 0}, z \in \mathbb{C}^{n}$ and $u, v \in C$.

Since $F_{0}$ satisfies estimate (1.4) with $\alpha=\frac{2}{n-1}$, therefore we conclude that

$$
\left|F_{m}(z, \sigma)\right| \leq C \sigma^{\frac{2}{n-1}},
$$

where $F_{m}=\psi_{m}, \partial_{x_{j}} \psi_{m}, \partial_{y_{j}} \psi_{m}, \sigma \partial_{\sigma} \psi_{m}(x, y, \sigma)$ with $1 \leq j \leq n$ and constant $C$ is independent of $m$.

In view of Duhamel's formula (see, Lemma A.1 in [6]) and in order to find solution for given IVP (1.1), (1.2) with initial value $f \in \tilde{W}_{\mathcal{L}}^{1,2}\left(\mathbb{C}^{n}\right)$, it is sufficient to find the solution of the following equation

$$
u(z, t)=e^{-i\left(t-t_{0}\right) \mathcal{L}} f(z)-i \int_{t_{0}}^{t} e^{-i(t-s) \mathcal{L}} G(z, u(z, s)) d s .
$$

In view of Banach fixed point theorem, for given $T>0, u: \mathbb{C}^{n} \times\left(t_{0}-T, t_{0}+\right.$ $T) \rightarrow \mathbb{C}$ and $m \geq 0$, we consider the operator $\mathcal{H}_{m}$ given by the following

$$
\mathcal{H}_{m}(u)(z, t)=e^{-i\left(t-t_{0}\right) \mathcal{L}} f(z)-i \int_{t_{0}}^{t} e^{-i(t-s) \mathcal{L}} G_{m}(z, u(z, s)) d s .
$$




\section{Some Auxilliary estimates}

Lemma 2.1. [Sobolev Embedding Theorem] We have the continuous inclusion

$$
\begin{array}{lll}
\tilde{W}_{\mathcal{L}}^{1, p_{1}}\left(\mathbb{C}^{n}\right) \hookrightarrow L^{p_{2}}\left(\mathbb{C}^{n}\right) & \text { for } p_{1} \leq p_{2} \leq \frac{2 n p_{1}}{2 n-p_{1}} & \text { if } p_{1}<2 n \\
& \text { for } p_{1} \leq p_{2}<\infty & \text { if } p_{1}=2 n \\
& \text { for } p_{1} \leq p_{2} \leq \infty & \text { if } p_{1}>2 n .
\end{array}
$$

Proof. Let $f \in \tilde{W}_{\mathcal{L}}^{1, p_{1}}\left(\mathbb{C}^{n}\right)$ and $\epsilon>0$. Consider $u_{\epsilon}=e^{-\epsilon \mathcal{L}} f$. Then $u_{\epsilon} \in$ $\tilde{W}_{\mathcal{L}}^{1, p_{1}}\left(\mathbb{C}^{n}\right) \cap C^{\infty}\left(\mathbb{C}^{n}\right)$ and we have

$$
2\left|u_{\epsilon}\right| \frac{\partial}{\partial x_{j}}\left|u_{\epsilon}\right|=\frac{\partial}{\partial x_{j}}\left(\overline{u_{\epsilon}} u_{\epsilon}\right)=2 \Re\left(\overline{u_{\epsilon}} \frac{\partial}{\partial x_{j}} u_{\epsilon}\right)=2 \Re\left(\overline{u_{\epsilon}}\left(\frac{\partial}{\partial x_{j}}-\frac{i y_{j}}{2}\right) u_{\epsilon}\right) .
$$

Hence on the set $A=\left\{z \in \mathbb{C}^{n} \mid u_{\epsilon}(z) \neq 0\right\}$, we have

$$
\left|\frac{\partial}{\partial x_{j}}\right| u_{\epsilon}||=\left|\Re\left(\frac{\overline{u_{\epsilon}}}{\left|u_{\epsilon}\right|}\left(\frac{\partial}{\partial x_{j}}-\frac{i y_{j}}{2}\right) u_{\epsilon}\right)\right| \leq \frac{1}{2}\left(\left|Z_{j} u_{\epsilon}\right|+\left|\bar{Z}_{j} u_{\epsilon}\right|\right) .
$$

Similarly $\left|\frac{\partial}{\partial y_{j}}\right| u_{\epsilon}|| \leq \frac{1}{2}\left(\left|Z_{j} u_{\epsilon}\right|+\left|\bar{Z}_{j} u_{\epsilon}\right|\right)$ on $A$. Note that $\left\|u_{\epsilon}\right\|_{L^{p_{2}\left(\mathbb{C}^{n}\right)}}=\left\|u_{\epsilon} \chi_{A}\right\|_{L^{p_{2}\left(\mathbb{C}^{n}\right)}}$.

By usual Sobolev embedding on $\mathbb{C}^{n}$ and above observations, we have inequality $\left\|u_{\epsilon}\right\|_{L^{p_{2}\left(\mathbb{C}^{n}\right)}} \leq C\left\|\mid u_{\epsilon} \chi_{A}\right\|_{W^{1, p_{1}}} \leq C\left\|u_{\epsilon}\right\|_{\tilde{W}_{\mathcal{L}}^{1, p_{1}}}$. Since $u_{\epsilon}=e^{-\epsilon \mathcal{L}} f \rightarrow f$ in $\tilde{W}_{\mathcal{L}}^{1, p_{1}}\left(\mathbb{C}^{n}\right)$ and also in $L^{p_{2}}\left(\mathbb{C}^{n}\right)$ as $\epsilon \rightarrow 0$ (see [7]), therefore we have $\|f\|_{L^{p_{2}\left(\mathbb{C}^{n}\right)}} \leq C\|f\|_{\tilde{W}_{\mathcal{L}}^{1, p_{1}\left(\mathbb{C}^{n}\right)}}$, where constant $C$ is a generic constant independent of $f$. Hence Lemma is proved.

Lemma 2.2. Let $u, v \in L^{\gamma}\left(I, \tilde{W}_{\mathcal{L}}^{1, \rho}\left(\mathbb{C}^{n}\right)\right)$ for some interval $I$, then following estimate holds for each $m \in \mathbb{Z}_{\geq 0}$

$$
\begin{array}{r}
\left\|G_{m}(z, u)-G_{m}(z, v)\right\|_{L^{\gamma^{\prime}}\left(I, L^{\rho^{\prime}}\left(\mathbb{C}^{n}\right)\right)} \leq C\|u-v\|_{L^{\gamma}\left(I, L^{\rho}\left(\mathbb{C}^{n}\right)\right)} \times \\
\left(\|u\|_{L^{\gamma}\left(I, \tilde{W}_{\mathcal{L}}^{1, \rho}\left(\mathbb{C}^{n}\right)\right)}+\|v\|_{L^{\gamma}\left(I, \tilde{W}_{\mathcal{L}}^{1, \rho}\left(\mathbb{C}^{n}\right)\right)}\right)^{\frac{2}{n-1}}
\end{array}
$$

where constant $C$ is independent of $u, v, m, t_{0}$ and $I$.

Proof. Since $\frac{1}{\rho^{\prime}}=\frac{1}{\rho}+\frac{n-1}{n^{2}}=\frac{1}{\rho}+\frac{2}{n-1} \cdot \frac{n-1}{n \gamma}$, by using Hölder's inequality in the $z$-variable in (1.9) and by embedding theorem (Lemma 2.1), we get for each $t \in I$

$$
\begin{aligned}
& \left\|G_{m}(\cdot, u(\cdot, t))-G_{m}(\cdot, v(\cdot, t))\right\|_{L^{\rho^{\prime}}\left(\mathbb{C}^{n}\right)} \\
\leq & C\|(u-v)(\cdot, t)\|_{L^{\rho}\left(\mathbb{C}^{n}\right)}\left(\|u(\cdot, t)\|_{L^{\frac{n \gamma}{n-1}\left(\mathbb{C}^{n}\right)}}+\|v(\cdot, t)\|_{L^{\frac{n \gamma}{n-1}\left(\mathbb{C}^{n}\right)}}\right)^{\frac{\gamma}{n}} \\
\leq & C\|(u-v)(\cdot, t)\|_{L^{\rho}\left(\mathbb{C}^{n}\right)}\left(\|u(\cdot, t)\|_{\tilde{W}_{\mathcal{L}}^{1, \rho}\left(\mathbb{C}^{n}\right)}+\|v(\cdot, t)\|_{\tilde{W}_{\mathcal{L}}^{1, \rho}\left(\mathbb{C}^{n}\right)}\right)^{\frac{\gamma}{n}} .
\end{aligned}
$$

Since $\frac{1}{\gamma^{\prime}}=\frac{1}{\gamma}+\frac{1}{n}$, by taking $L^{\gamma^{\prime}}$ norm in $t$-variable in this inequality and then by using Höder's inequality we get desired estimate (2.1). 
Lemma 2.3. Let $I$ be a bounded interval and $u \in L^{\infty}\left(I, \tilde{W}_{\mathcal{L}}^{1,2}\left(\mathbb{C}^{n}\right)\right) \cap L^{\gamma}$ $\left(I, \tilde{W}_{\mathcal{L}}^{1, \rho}\left(\mathbb{C}^{n}\right)\right)$, then following estimate holds

$$
\begin{gathered}
\left\|G_{m}(z, u(z, t))-G(z, u(z, t))\right\|_{L^{\gamma^{\prime}}\left(I, L^{\rho^{\prime}}\left(\mathbb{C}^{n}\right)\right)} \\
\leq C|I|^{\frac{n-1}{2 n}} m^{-\frac{1}{n(n-1)}\|u\|_{L^{\infty}\left(I, \tilde{W}_{\mathcal{L}}^{1,2}\left(\mathbb{C}^{n}\right)\right)}^{\frac{n^{2}-n+1}{n(n-1)}}\|u\|_{L^{\gamma}\left(I, \tilde{W}_{\mathcal{L}}^{1, \rho}\left(\mathbb{C}^{n}\right)\right)}^{\frac{2}{n-1}}}
\end{gathered}
$$

for all $m \geq 1$, where constant $C$ is independent of $m, u$ and $I$.

Proof. Note that

$$
G_{m}(z, u(z, t))-G(z, u(z, t))=\left(u \chi_{|u(z, t)|>m}(z, t)\right)\left(\psi_{m}(z,|u|)-\psi(z,|u|)\right) .
$$

Therefore $\left|G_{m}(z, u(z, t))-G(z, u(z, t))\right| \leq C\left|u \chi_{|u(z, t)|>m}(z, t)\right||u|^{\frac{2}{n-1}}$. By Taking $L^{\rho^{\prime}}$-norm in the $z$-variable, we have

$$
\begin{aligned}
\left\|G_{m}(z, u)-G(z, u)\right\|_{L^{\rho^{\prime}}\left(\mathbb{C}^{n}\right)} & \leq C\left\|u \chi_{|u|>m}(\cdot, t)\right\|_{L^{\rho}\left(\mathbb{C}^{n}\right)}\|u(\cdot, t)\|_{L^{\frac{n \gamma}{n-1}\left(\mathbb{C}^{n}\right)}}^{\frac{\gamma}{n}} \\
& \leq C\left\|u \chi_{|u|>m}(\cdot, t)\right\|_{L^{\rho}\left(\mathbb{C}^{n}\right)}\|u(\cdot, t)\|_{\tilde{W}_{\mathcal{L}}^{1, \rho}\left(\mathbb{C}^{n}\right)}^{\frac{\gamma}{n}} .
\end{aligned}
$$

Now we observe the following

$$
\begin{aligned}
\left\|u \chi_{|u|>m}(\cdot, t)\right\|_{L^{\rho}\left(\mathbb{C}^{n}\right)}^{\rho} & =\int_{\mathbb{C}^{n}}|u|^{\rho} \chi_{|u|>m}(z, t) d z \\
& \leq \int_{\mathbb{C}^{n}} m^{-\frac{\rho}{n(n-1)}}|u|^{\frac{2 n}{n-1}} d z \\
& \leq m^{-\frac{\rho}{n(n-1)}\|u\|_{\frac{\left(n^{2}-n+1\right) \rho}{n(n-1)}}^{\frac{2 n}{n^{n-1}\left(\mathbb{C}^{n}\right)}}} \\
& \leq m^{-\frac{\rho}{n(n-1)}\|u\|_{\tilde{W}_{\mathcal{L}}^{1,2}\left(\mathbb{C}^{n}\right)}^{\frac{\left(n^{2}-n+1\right) \rho}{n(n-1)}}} \\
\left\|u \chi_{|u|>m}(\cdot, t)\right\|_{L^{\rho}} & \leq m^{-\frac{1}{n(n-1)}\|u\|_{\tilde{W}_{\mathcal{L}}^{1,2}\left(\mathbb{C}^{n}\right)}^{\frac{\left(n^{2}-n+1\right)}{n-1)}}}
\end{aligned}
$$

By taking $L^{\gamma}$-norm in the $t$-variable we have

$$
\left\|u \chi_{|u|>m}\right\|_{L^{\gamma}\left(I, L^{\rho}\left(\mathbb{C}^{n}\right)\right)} \leq|I|^{\frac{n-1}{2 n}} m^{-\frac{1}{n(n-1)}}\|u\|_{L^{\infty}\left(I, \tilde{W}_{\mathcal{L}}^{1,2}\left(\mathbb{C}^{n}\right)\right)}^{\frac{\left(n^{2}-n+1\right)}{n(n-1)}} .
$$

By taking $L^{\gamma^{\prime}}$-norm in the $t$-variable in estimate (2.3) and using Hölder's inequality, we get

$$
\left\|G_{m}(z, u)-G(z, u)\right\|_{L^{\gamma^{\prime}}\left(I, L^{\rho^{\prime}}\right)} \leq C\left\|u \chi_{|u|>m}\right\|_{L^{\gamma}\left(I, L^{\rho}\right)}\|u\|_{L^{\gamma}\left(I, \tilde{W}_{\mathcal{L}}^{1, \rho}\right)}^{\frac{2}{n-1}} .
$$

By using inequality (2.4) in the above inequality, we get the desired estimate. 
Lemma 2.4. Let $u \in L^{\gamma}\left(I, \tilde{W}_{\mathcal{L}}^{1, \rho}\left(\mathbb{C}^{n}\right)\right)$ for some interval $I$. Then for each $m \in \mathbb{Z}_{\geq 0}, G_{m}(z, u(z, t)) \in L^{\gamma^{\prime}}\left(I, \tilde{W}_{\mathcal{L}}^{1, \rho^{\prime}}\left(\mathbb{C}^{n}\right)\right)$ and following estimates hold:

$$
\begin{aligned}
\left\|S G_{m}(z, u(z, t))\right\|_{L^{\gamma^{\prime}}\left(I, L^{\rho^{\prime}}\left(\mathbb{C}^{n}\right)\right)} & \leq C\|u\|_{L^{\gamma}\left(I, \tilde{W}_{\mathcal{L}}^{1, \rho}\left(\mathbb{C}^{n}\right)\right)}^{\frac{n+1}{n-1}} \\
\left\|G_{m}(z, u(z, t))\right\|_{L^{\gamma^{\prime}}\left(I, \tilde{W}_{\mathcal{L}}^{1, \rho^{\prime}}\left(\mathbb{C}^{n}\right)\right)} & \leq C\|u\|_{L^{\gamma}\left(I, \tilde{W}_{\mathcal{L}}^{1, \rho}\left(\mathbb{C}^{n}\right)\right)}^{\frac{n+1}{n-1}}
\end{aligned}
$$

where $S=Z_{j}, \bar{Z}_{j}$ or $I d, 1 \leq j \leq n$ and constant $C$ is independent of $u$ and $I$. Proof. Since $\psi_{m}, \partial_{x_{j}} \psi_{m}, \partial_{y_{j}} \psi_{m},|u| \partial_{|u|} \psi_{m}$ satisfy estimate (1.10), therefore we have

$$
\left|S G_{m}(z, u)\right| \leq C|u|^{\frac{2}{n-1}}\left(|u|+\left|Z_{j} u\right|+\left|\bar{Z}_{j} u\right|\right)
$$

where $S=Z_{j}, \bar{Z}_{j}(1 \leq j \leq n)$ or $I d$, see Lemma 3.4 in [7]. Now estimate (2.5]) follows from Hölder's inequality and embedding theorem (Lemma 2.1) as we used in the proof of Lemma 2.2. Estimate (2.6) is a consequence of estimate (2.5).

Proposition 2.5. Let $I$ be a bounded interval such that $t_{0} \in \bar{I}$.

(i): If $u, v \in L^{\gamma}\left(I, \tilde{W}_{\mathcal{L}}^{1, \rho}\left(\mathbb{C}^{n}\right)\right)$, then $\mathcal{H}_{m} u-\mathcal{H}_{m} v \in L^{q}\left(I, L^{p}\left(\mathbb{C}^{n}\right)\right)$ for every admissible pair $(q, p)$, for every $m \in \mathbb{Z}_{\geq 0}$ and following estimate holds:

$$
\begin{aligned}
& \left\|\mathcal{H}_{m} u-\mathcal{H}_{m} v\right\|_{L^{q}\left(I, L^{p}\left(\mathbb{C}^{n}\right)\right)} \\
\leq & C\|u-v\|_{L^{\gamma}\left(I, L^{\rho}\left(\mathbb{C}^{n}\right)\right)}\left(\|u\|_{L^{\gamma}\left(I, \tilde{W}_{\mathcal{L}}^{1, \rho}\left(\mathbb{C}^{n}\right)\right)}+\|v\|_{L^{\gamma}\left(I, \tilde{W}_{\mathcal{L}}^{1, \rho}\left(\mathbb{C}^{n}\right)\right)}\right)^{\frac{2}{n-1}} .
\end{aligned}
$$

(ii): If $u \in L^{\infty}\left(I, \tilde{W}_{\mathcal{L}}^{1,2}\left(\mathbb{C}^{n}\right)\right) \cap L^{\gamma}\left(I, \tilde{W}_{\mathcal{L}}^{1, \rho}\left(\mathbb{C}^{n}\right)\right)$, then $\mathcal{H}_{m} u-\mathcal{H} u \in$ $L^{q}\left(I, L^{p}\left(\mathbb{C}^{n}\right)\right)$ for every admissible pair $(q, p)$, for every $m \in \mathbb{Z}_{\geq 1}$ and following estimate holds

$$
\begin{aligned}
& \left\|\mathcal{H}_{m} u-\mathcal{H} u\right\|_{L^{q}\left(I, L^{p}\left(\mathbb{C}^{n}\right)\right)} \\
\leq & C|I|^{\frac{n-1}{2 n}} m^{-\frac{1}{n(n-1)}}\|u\|_{L^{\infty}\left(I, \tilde{W}_{\mathcal{L}}^{1,2}\left(\mathbb{C}^{n}\right)\right)}^{\frac{n^{2}-n+1}{n(n-1)}}\|u\|_{L^{\gamma}\left(I, \tilde{W}_{\mathcal{L}}^{1, \rho}\left(\mathbb{C}^{n}\right)\right)}^{\frac{2}{n-1}}
\end{aligned}
$$

where constant $C$ is independent of $u, v, m$ and $t_{0}$.

Proof. Estimate (2.7) follows from Strichartz estimates (Theorem 3.3 in [7]) and Lemma 2.2, whereas estimate (2.8) follows from Strichartz estimates and Lemma 2.3 .

Now we state the following Proposition, which is useful in proving stability. 
Proposition 2.6. Let $\Phi$ be a continuous complex valued function on $\mathbb{C}$ such that $|\Phi(w)| \leq C|w|^{\frac{2}{n-1}}$ with $n \geq 2$. Let $\left\{u_{m}\right\}$ be a bounded sequence in $L^{\gamma}\left(I, \tilde{W}_{\mathcal{L}}^{1, \rho}\left(\mathbb{C}^{n}\right)\right)$ for some interval $I$. If $u_{m} \rightarrow u$ in $L^{\gamma}\left(I, L^{\rho}\left(\mathbb{C}^{n}\right)\right)$ then $u \in$ $L^{\gamma}\left(I, \tilde{W}_{\mathcal{L}}^{1, \rho}\left(\mathbb{C}^{n}\right)\right)$ and $\left[\Phi\left(u_{m}\right)-\Phi(u)\right] S u \rightarrow 0$ in $L^{\gamma^{\prime}}\left(I, L^{\rho^{\prime}}\left(\mathbb{C}^{n}\right)\right)$, for $S=$ Id, $Z_{j}, \bar{Z}_{j} ; 1 \leq j \leq n$.

Proof. First we will prove $u \in L^{\gamma}\left(I, \tilde{W}_{\mathcal{L}}^{1, \rho}\left(\mathbb{C}^{n}\right)\right)$. By duality argument (also see Lemma A.2.1 in [4]), we have

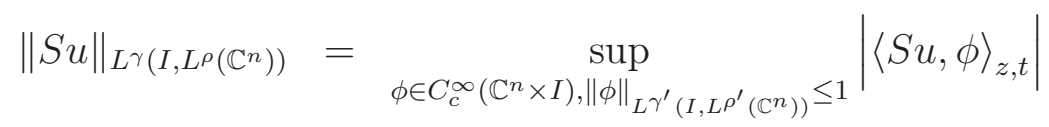

$$
\begin{aligned}
& =\sup _{\phi}\left|\left\langle u, S^{*} \phi\right\rangle_{z, t}\right| \\
& =\sup _{\phi} \lim _{m \rightarrow \infty}\left|\left\langle u_{m}, S^{*} \phi\right\rangle_{z, t}\right| \\
& =\sup _{\phi} \lim _{m \rightarrow \infty}\left|\left\langle S u_{m}, \phi\right\rangle_{z, t}\right| \\
& \leq \sup _{\phi} \liminf _{m \rightarrow \infty}\left\|S u_{m}\right\|_{L^{\gamma}\left(I, L^{\rho}\left(\mathbb{C}^{n}\right)\right)}\|\phi\|_{L^{\gamma^{\prime}}\left(I, L^{\rho^{\prime}}\left(\mathbb{C}^{n}\right)\right)} \\
& \leq \liminf _{m \rightarrow \infty}\left\|S u_{m}\right\|_{L^{\gamma}\left(I, L^{\rho}\left(\mathbb{C}^{n}\right)\right)}
\end{aligned}
$$

for $S=Z_{j}, \bar{Z}_{j} ; 1 \leq j \leq n$. Therefore

$$
\|u\|_{L^{\gamma\left(I, \tilde{W}_{\mathcal{L}}^{1, \rho}\left(\mathbb{C}^{n}\right)\right)}} \leq \liminf _{m \rightarrow \infty}\left\|u_{m}\right\|_{L^{\gamma\left(I, \tilde{W}_{\mathcal{L}}^{1, \rho}\left(\mathbb{C}^{n}\right)\right)}}<\infty .
$$

Since $u_{m} \rightarrow u$ in $L^{\gamma}\left(I, L^{\rho}\left(\mathbb{C}^{n}\right)\right)$, we can extract a subsequence still denoted by $u_{k}$ such that

$$
\left\|u_{k+1}-u_{k}\right\|_{L^{\gamma}\left(I, L^{\rho}\left(\mathbb{C}^{n}\right)\right)} \leq \frac{1}{2^{k}}
$$

for all $k \geq 1$ and $u_{k}(z, t) \rightarrow u(z, t)$ a.e. Hence by continuity of $\Phi$,

$$
\left[\Phi\left(u_{k}\right)-\Phi(u)\right] S u \rightarrow 0 \text { for a.e }(z, t) \in \mathbb{C}^{n} \times I .
$$

We establish the norm convergence by appealing to a dominated convergence argument in $z$ and $t$ variables successively.

Consider the function $H(z, t)=\sum_{k=1}^{\infty}\left|u_{k+1}(z, t)-u_{k}(z, t)\right|$. Clearly $H \in$ $L^{\gamma}\left(I, L^{\rho}\left(\mathbb{C}^{n}\right)\right)$. Also for $l>k,\left|\left(u_{l}-u_{k}\right)(z, t)\right| \leq\left|u_{l}-u_{l-1}\right|+\cdots+\left|u_{k+1}-u_{k}\right| \leq$ $H(z, t)$, hence $\left|u_{k}-u\right| \leq H$. This leads to the pointwise almost everywhere inequality

$$
\left|u_{k}(z, t)\right| \leq|u(z, t)|+H(z, t)=v(z, t)
$$

Hence

$$
\left|\left[\Phi\left(u_{k}\right)-\Phi(u)\right] S u(z, t)\right|^{\rho^{\prime}} \leq C\left[v^{\frac{2}{n-1}}+|u|^{\frac{2}{n-1}}\right]^{\rho^{\prime}}|S u(z, t)|^{\rho^{\prime}} .
$$


Since $u, v \in L^{\gamma}\left(I, L^{\rho}\left(\mathbb{C}^{n}\right)\right)$, using Hölder's inequality with $\frac{1}{\rho^{\prime}}=\frac{1}{\rho}+\frac{n-1}{n^{2}}=$ $\frac{1}{\rho}+\frac{2}{n-1} \cdot \frac{n-1}{n \gamma}$ and Lemma 2.1, we get

$$
\begin{aligned}
& \int_{\mathbb{C}^{n}}\left[v^{\frac{2}{n-1}}+|u|^{\frac{2}{n-1}}\right]^{\rho^{\prime}}|S u(z, t)|^{\rho^{\prime}} d z \\
& \leq\left(\|v(\cdot, t)\|_{L^{\frac{n \gamma}{n-1}\left(\mathbb{C}^{n}\right)}}+\|u(\cdot, t)\|_{L^{\frac{n \gamma}{n-1}\left(\mathbb{C}^{n}\right)}}\right)^{\frac{\rho^{\prime} \gamma}{n}}\|S u(\cdot, t)\|_{L^{\rho}\left(\mathbb{C}^{n}\right)}^{\rho^{\prime}} \\
& \leq\left(\|v(\cdot, t)\|_{\tilde{W}_{\mathcal{L}}^{1, \rho}\left(\mathbb{C}^{n}\right)}+\|u(\cdot, t)\|_{\tilde{W}_{\mathcal{L}}^{1, \rho}\left(\mathbb{C}^{n}\right)}\right)^{\frac{\rho^{\prime} \gamma}{n}}\|S u(\cdot, t)\|_{\left.L^{\rho} \mathbb{C}^{\prime}\right)}^{\rho^{\prime}}<\infty
\end{aligned}
$$

for a.e. $t \in I$. Thus in view of (2.11), (2.12) and using dominated convergence theorem in the $z$-variable, we see that

$$
\left\|\left[\Phi\left(u_{k}\right)-\Phi(u)\right] S u(\cdot, t)\right\|_{L^{p^{\prime}}\left(\mathbb{C}^{n}\right)} \rightarrow 0
$$

as $k \rightarrow \infty$, for a.e. $t$.

Again, in view of (2.11) and (2.12), we get

$$
\begin{aligned}
& \left\|\left[\Phi\left(u_{k}\right)-\Phi(u)\right] S u(\cdot, t)\right\|_{L^{\rho^{\prime}}\left(\mathbb{C}^{n}\right)} \\
& \leq C\left(\|v(\cdot, t)\|_{\tilde{W}_{\mathcal{L}}^{1, \rho}\left(\mathbb{C}^{n}\right)}+\|u(\cdot, t)\|_{\tilde{W}_{\mathcal{L}}^{1, \rho}\left(\mathbb{C}^{n}\right)}\right)^{\frac{\gamma}{n}}\|S u(\cdot, t)\|_{L^{\rho}\left(\mathbb{C}^{n}\right)} .
\end{aligned}
$$

Since $\frac{1}{\gamma^{\prime}}=\frac{1}{\gamma}+\frac{1}{n}$, an application of the Hölder's inequality in the $t$-variable shows that

$$
\begin{aligned}
& \left\|\left[\Phi\left(u_{k}\right)-\Phi(u)\right] S u\right\|_{L^{\gamma^{\prime}}\left(I, L^{\rho^{\prime}}\left(\mathbb{C}^{n}\right)\right)} \\
& \leq C\left(\|v\|_{L^{\gamma}\left(I, \tilde{W}_{\mathcal{L}}^{1, \rho}\left(\mathbb{C}^{n}\right)\right)}+\|u\|_{L^{\gamma}\left(I, \tilde{W}_{\mathcal{L}}^{1, \rho}\left(\mathbb{C}^{n}\right)\right)}\right)^{\frac{\gamma}{n}}\|S u\|_{L^{\gamma}\left(I, L^{\rho}\left(\mathbb{C}^{n}\right)\right)} .
\end{aligned}
$$

Hence a further application of dominated convergence theorem with (2.13) shows that $\left\|\left(\Phi\left(u_{k}\right)-\Phi(u)\right) S u\right\|_{L^{\gamma^{\prime}\left(I, L^{\rho^{\prime}}\right)}} \rightarrow 0$, as $k \rightarrow \infty$.

Thus we have shown that $\left[\Phi\left(u_{m_{k}}\right)-\Phi(u)\right] S u \rightarrow 0$ in $L^{\gamma^{\prime}}\left(I, L^{\rho^{\prime}}\left(\mathbb{C}^{n}\right)\right)$ for some subsequence $u_{m_{k}}$ whenever $u_{m} \rightarrow u$ in $L^{\gamma}\left(I, L^{\rho}\left(\mathbb{C}^{n}\right)\right)$. But the above arguments are also valid if we had started with any subsequence of $u_{m}$. It follows that any subsequence of $\left[\Phi\left(u_{m}\right)-\Phi(u)\right] S u$ has a subsequence that converges to 0 in $L^{\gamma^{\prime}}\left(I, L^{\rho^{\prime}}\left(\mathbb{C}^{n}\right)\right)$. From this we conclude that the original sequence $\left[\Phi\left(u_{m}\right)-\Phi(u)\right] S u$ converges to zero in $L^{\gamma^{\prime}}\left(I, L^{\rho^{\prime}}\left(\mathbb{C}^{n}\right)\right)$, hence the proposition.

\section{Proof of Theorem 1.1}

Proof. (of Theorem 1.1): (Local existence): Since $G_{m}(z, \cdot): \mathbb{C} \rightarrow \mathbb{C}$ is globally lipschitz for each $m \geq 1$, see (1.8), therefore from [7] it follows that there exists a unique global solution $u_{m} \in C\left(\mathbb{R}, \tilde{W}_{\mathcal{L}}^{1,2}\left(\mathbb{C}^{n}\right)\right)$ of the initial value problem

$$
\begin{aligned}
i \partial_{t} v(z, t)-\mathcal{L} v(z, t) & =G_{m}(z, v), z \in \mathbb{C}^{n}, t \in \mathbb{R} \\
v\left(\cdot, t_{0}\right) & =f .
\end{aligned}
$$


Furthermore $\mathcal{H}_{m} u_{m}=u_{m}$ and $u_{m} \in L_{\text {loc }}^{q}\left(\mathbb{R}, \tilde{W}_{\mathcal{L}}^{1, p}\left(\mathbb{C}^{n}\right)\right)$ for every admissible pair $(q, p)$. We deduce from Lemma 2.4 and Strichartz estimates (Theorem 3.3 in [7]) that

$$
\begin{aligned}
& \left\|u_{m}\right\|_{L^{q}\left(\left(t_{0}, t_{0}+T\right), \tilde{W}_{\mathcal{L}}^{1, p}\left(\mathbb{C}^{n}\right)\right)} \\
& \leq\left\|e^{-i\left(t-t_{0}\right) \mathcal{L}} f\right\|_{L^{q}\left(\left(t_{0}, t_{0}+T\right), \tilde{W}_{\mathcal{L}}^{1, p}\left(\mathbb{C}^{n}\right)\right)}+C\left\|u_{m}\right\|_{L^{\gamma}\left(\left(t_{0}, t_{0}+T\right), \tilde{W}_{\mathcal{L}}^{1, \rho}\left(\mathbb{C}^{n}\right)\right)}^{\frac{n+1}{n-1}} .
\end{aligned}
$$

Let $l \geq m$, we see that

$$
u_{m}-u_{l}=\left(\mathcal{H}_{m}\left(u_{m}\right)-\mathcal{H}_{m}\left(u_{l}\right)\right)+\left(\mathcal{H}_{m}\left(u_{l}\right)-\mathcal{H}\left(u_{l}\right)\right)+\left(\mathcal{H}\left(u_{l}\right)-\mathcal{H}_{l}\left(u_{l}\right)\right) .
$$

From Proposition 2.5, we deduce that

$$
\begin{array}{r}
\left\|u_{m}-u_{l}\right\|_{L^{q}\left(\left(t_{0}, t_{0}+T\right), L^{p}\left(\mathbb{C}^{n}\right)\right)} \leq C\left(\left\|u_{m}\right\|_{L^{\gamma}\left(\left(t_{0}, t_{0}+T\right), \tilde{W}_{\mathcal{L}}^{1, \rho}\right)}+\left\|u_{l}\right\|_{L^{\gamma}\left(\left(t_{0}, t_{0}+T\right), \tilde{W}_{\mathcal{L}}^{1, \rho}\right)}\right)^{\frac{2}{n-1}} \times \\
(3.4) \quad\left(\left\|u_{m}-u_{l}\right\|_{L^{\gamma}\left(\left(t_{0}, t_{0}+T\right), L^{\rho}\right)}+T^{\frac{n-1}{2 n}} m^{-\frac{1}{n(n-1)}}\left\|u_{l}\right\|_{L^{\infty}\left(\left(t_{0}, t_{0}+T\right), \tilde{W}_{\mathcal{L}}^{1,2}\right)}^{\frac{n^{2}-n+1}{n(n-1)}}\right) .
\end{array}
$$

We choose $T \leq \pi$, therefore we can take constant $C$ to be independent of $T$. Let $\tilde{C}$ be larger than the constant $C$ that appear in (3.3), (3.4), (2.7), (2.8) and in Strichartz estimates for the particular choice of the admissible pair $(q, p)=(\gamma, \rho)$. Fixed $\delta$ small enough so that

$$
\tilde{C}(4 \delta)^{\frac{2}{n-1}}<\frac{1}{2}
$$

We claim that if $0<T \leq \pi$ is such that

$$
\left\|e^{-i\left(t-t_{0}\right) \mathcal{L}} f\right\|_{L^{\gamma}\left(\left(t_{0}, t_{0}+T\right), \tilde{W}_{\mathcal{L}}^{1, \rho}\left(\mathbb{C}^{n}\right)\right)} \leq \delta
$$

then

$$
\begin{aligned}
& \sup _{m \geq 1}\left\|u_{m}\right\|_{L^{\gamma}\left(\left(t_{0}, t_{0}+T\right), \tilde{W}_{\mathcal{L}}^{1, \rho}\left(\mathbb{C}^{n}\right)\right)} \leq 2 \delta \\
& \sup _{m \geq 1}\left\|u_{m}\right\|_{L^{q}\left(\left(t_{0}, t_{0}+T\right), \tilde{W}_{\mathcal{L}}^{1, p}\left(\mathbb{C}^{n}\right)\right)}<\infty
\end{aligned}
$$

for every admissible pair $(q, p)$. Let $\theta_{m}(t)=\left\|u_{m}\right\|_{L^{\gamma}\left(\left(t_{0}, t_{0}+t\right), \tilde{W}_{\mathcal{L}}^{1, \rho}\left(\mathbb{C}^{n}\right)\right)}$. From (3.3), we see that

$$
\theta_{m}(t) \leq \delta+\tilde{C} \theta_{m}(t)^{\frac{n+1}{n-1}} .
$$

If $\theta_{m}(t)=2 \delta$ for some $t \in\left(t_{0}, t_{0}+T\right]$, then

$$
2 \delta \leq \delta+\tilde{C}(2 \delta)^{\frac{n+1}{n-1}}<2 \delta
$$

which is a contradiction. Since $\theta_{m}$ is a continuous function with $\theta_{m}\left(t_{0}\right)=0$ therefore we conclude that $\theta_{m}(t)<2 \delta$ for all $t \in\left(t_{0}, t_{0}+T\right]$, which proves 
(3.7). From (3.3), we see that

$$
\begin{aligned}
\sup _{m}\left\|u_{m}\right\|_{L^{q}\left(\left(t_{0}, t_{0}+T\right), \tilde{W}_{\mathcal{L}}^{1, p}\left(\mathbb{C}^{n}\right)\right)} & \leq\left\|e^{-i\left(t-t_{0}\right) \mathcal{L}} f\right\|_{L^{q}\left(\left(t_{0}, t_{0}+T\right), \tilde{W}_{\mathcal{L}}^{1, p}\left(\mathbb{C}^{n}\right)\right)}+C(2 \delta)^{\frac{n+1}{n-1}} \\
& \leq C(q, p, n, \delta, f)<\infty .
\end{aligned}
$$

This proves (3.8). Put $(q, p)=(\gamma, \rho)$ in (3.4), we see that

$$
\begin{aligned}
\left\|u_{m}-u_{l}\right\|_{L^{\gamma}\left(\left(t_{0}, t_{0}+T\right), L^{\rho}\left(\mathbb{C}^{n}\right)\right)} & \leq \frac{1}{2}\left(\left\|u_{m}-u_{l}\right\|_{L^{\gamma}\left(\left(t_{0}, t_{0}+T\right), L^{\rho}\left(\mathbb{C}^{n}\right)\right)}+C T^{\frac{n-1}{2 n}} m^{-\frac{1}{n(n-1)}}\right) \\
& \leq 2 C T^{\frac{n-1}{2 n}} m^{-\frac{1}{n(n-1)}} \rightarrow 0 \text { as } m \rightarrow \infty .
\end{aligned}
$$

This shows that $u_{m}$ is a cauchy sequence in $L^{\gamma}\left(\left(t_{0}, t_{0}+T\right), L^{\rho}\left(\mathbb{C}^{n}\right)\right)$ and from (3.4) it is also cauchy in $L^{q}\left(\left(t_{0}, t_{0}+T\right), L^{p}\left(\mathbb{C}^{n}\right)\right)$ for every admissible pair $(q, p)$. Let $u$ be its limit, then $u_{m} \rightarrow u$ in $L^{q}\left(\left(t_{0}, t_{0}+T\right), L^{p}\left(\mathbb{C}^{n}\right)\right)$ for every admissible pair $(q, p)$. By duality argument (see (2.9)) and from estimates (3.7), (3.8), we have

$$
\begin{aligned}
\|u\|_{L^{\gamma}\left(\left(t_{0}, t_{0}+T\right), \tilde{W}_{\mathcal{L}}^{1, \rho}\left(\mathbb{C}^{n}\right)\right)} & \leq 2 \delta \\
\|u\|_{L^{q}\left(\left(t_{0}, t_{0}+T\right), \tilde{W}_{\mathcal{L}}^{1, p}\left(\mathbb{C}^{n}\right)\right)} & <\infty .
\end{aligned}
$$

From Lemma 2.4, $G_{m}(z, u(z, t)) \in L^{\gamma^{\prime}}\left(\left(t_{0}, t_{0}+T\right), \tilde{W}_{\mathcal{L}}^{1, \rho^{\prime}}\left(\mathbb{C}^{n}\right)\right)$ for each $m \geq$ 0. From Strichartz estimates (Theorem 3.3 in [7]) and (1.11), $\mathcal{H} u \in L^{q}\left(\left(t_{0}, t_{0}+\right.\right.$ $\left.T), \tilde{W}_{\mathcal{L}}^{1, p}\left(\mathbb{C}^{n}\right)\right)$ for every admissible pair $(q, p)$.

From Lemma $2.2\left\|G_{m}\left(z, u_{m}\right)-G_{m}(z, u)\right\|_{L^{\gamma^{\prime}}\left(\left(t_{0}, t_{0}+T\right), L^{\rho^{\prime}}\left(\mathbb{C}^{n}\right)\right)} \rightarrow 0$ and from

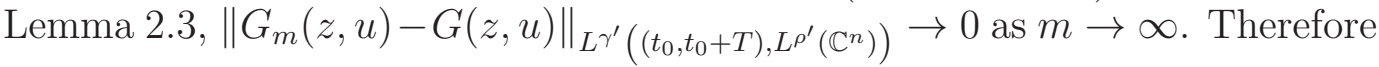

$$
\left\|G_{m}\left(z, u_{m}\right)-G(z, u)\right\|_{L^{\gamma^{\prime}}\left(\left(t_{0}, t_{0}+T\right), L^{\rho^{\prime}}\right)} \rightarrow 0 \text { as } m \rightarrow \infty .
$$

Since $u_{m}=\mathcal{H}_{m} u_{m}$ for each $m \geq 1$, from Strichartz estimates we deduce that

$$
\begin{aligned}
\left\|u_{m}-\mathcal{H} u\right\|_{L^{q}\left(\left(t_{0}, t_{0}+T\right), L^{p}\left(\mathbb{C}^{n}\right)\right)} & =\left\|\mathcal{H}_{m} u_{m}-\mathcal{H} u\right\|_{L^{q}\left(\left(t_{0}, t_{0}+T\right), L^{p}\left(\mathbb{C}^{n}\right)\right)} \\
& \leq C\left\|G_{m}\left(z, u_{m}\right)-G(z, u)\right\|_{L^{\gamma^{\prime}}\left(\left(t_{0}, t_{0}+T\right), L^{\rho^{\prime}}\right)} \rightarrow 0
\end{aligned}
$$

as $m \rightarrow \infty$. Therefore for $t \in\left(t_{0}, t_{0}+T\right)$

$$
u=\mathcal{H} u=e^{-i\left(t-t_{0}\right) \mathcal{L}} f(z)-i \int_{t_{0}}^{t} e^{-i(t-s) \mathcal{L}} G(z, u(z, s)) d s .
$$

From Strichartz estimates and estimate (3.10), $u \in C\left(\left[t_{0}, t_{0}+T\right], \tilde{W}_{\mathcal{L}}^{1,2}\right) \cap$ $L^{q}\left(\left(t_{0}, t_{0}+T\right), \tilde{W}_{\mathcal{L}}^{1, p}\left(\mathbb{C}^{n}\right)\right)$ for every admissible pair $(q, p)$. In view of Lemma A.1 in [6], $u$ is also a solution to the initial value problem (1.1), (1.2). Similarly solution exists on the interval $\left[t_{0}-T^{\prime}, t_{0}\right]$ for some $T^{\prime}>0$. Now we continue this process with initial time $t_{0}+T$ and $t_{0}-T^{\prime}$. In this way we construct maximal solution $u \in C\left(\left(T_{*}, T^{*}\right), \tilde{W}_{\mathcal{L}}^{1,2}\right) \cap L_{\text {loc }}^{q}\left(\left(T_{*}, T^{*}\right), \tilde{W}_{\mathcal{L}}^{1, p}\left(\mathbb{C}^{n}\right)\right)$ for every admissible pair $(q, p)$. 
Conservation of charge and energy: From [7], we have following conservation laws

$$
\begin{aligned}
& \left\|u_{m}(\cdot, t)\right\|_{L^{2}\left(\mathbb{C}^{n}\right)}=\|f\|_{L^{2}\left(\mathbb{C}^{n}\right)}, t \in \mathbb{R} \\
& E_{m}\left(u_{m}(\cdot, t)\right)=E_{m}(f), t \in \mathbb{R}
\end{aligned}
$$

where

$$
\begin{aligned}
E_{m}(f) & =\frac{1}{4} \sum_{j=1}^{n}\left(\left\|Z_{j} f\right\|_{L^{2}\left(\mathbb{C}^{n}\right)}^{2}+\left\|\bar{Z}_{j} f\right\|_{L^{2}\left(\mathbb{C}^{n}\right)}^{2}\right)+\int_{\mathbb{C}^{n}} \tilde{G_{m}}(z,|f(z)|) d z \\
\tilde{G_{m}}(z, \sigma) & =\int_{s=0}^{\sigma} G_{m}(z, s) d s, \sigma>0 .
\end{aligned}
$$

Since $u_{m} \rightarrow u$ in $L^{\infty}\left(\left[t_{0}-T, t_{0}+T\right], L^{2}\left(\mathbb{C}^{n}\right)\right)$ for sufficiently small $T>0$, therefore by taking limit $m \rightarrow \infty$ in (3.12), we get

$$
\|u(\cdot, t)\|_{L^{2}\left(\mathbb{C}^{n}\right)}=\|f\|_{L^{2}\left(\mathbb{C}^{n}\right)}, t \in\left[t_{0}-T, t_{0}+T\right] .
$$

By repeating this argument for any point in $\left(T_{*}, T^{*}\right)$, instead of $t_{0}$, we get conservation of charge on $\left(T_{*}, T^{*}\right)$.

Now we will prove conservation of energy. From (3.8), for each $t \in\left(t_{0}-\right.$ $\left.T, t_{0}+T\right)$, sequence $\left\|u_{m}(\cdot, t)\right\|_{\tilde{W}_{\mathcal{L}}^{1,2}\left(\mathbb{C}^{n}\right)}$ is uniformly bounded and $u_{m}(\cdot, t) \rightarrow$ $u(\cdot, t)$ in $L^{2}\left(\mathbb{C}^{n}\right)$, therefore by duality argument (see (2.9)), we have

$\sum_{j=1}^{n}\left(\left\|Z_{j} u(\cdot, t)\right\|_{L^{2}}^{2}+\left\|\bar{Z}_{j} u(\cdot, t)\right\|_{L^{2}}^{2}\right) \leq \liminf _{m \rightarrow \infty} \sum_{j=1}^{n}\left(\left\|Z_{j} u_{m}(\cdot, t)\right\|_{L^{2}}^{2}+\left\|\bar{Z}_{j} u_{m}(\cdot, t)\right\|_{L^{2}}^{2}\right)$.

Since $\left|\tilde{G_{m}}(z,|f(z)|)\right| \leq C|f|^{\frac{2 n}{n-1}}, f \in \tilde{W}_{\mathcal{L}}^{1,2}\left(\mathbb{C}^{n}\right) \subset L^{\frac{2 n}{n-1}}\left(\mathbb{C}^{n}\right)$, therefore by dominated convergence theorem, $\int_{\mathbb{C}^{n}} \tilde{G_{m}}(z,|f(z)|) d z \rightarrow \int_{\mathbb{C}^{n}} \tilde{G}(z,|f(z)|) d z$ as $m \rightarrow \infty$. Since $u_{m} \rightarrow u$ in $L^{q}\left(\left(t_{0}-T, t_{0}+T\right), L^{p}\left(\mathbb{C}^{n}\right)\right)$ for every admissible pair, therefore after choosing a suitable subsequence, we have pointwise convergence $u_{m}(z, t) \rightarrow u(z, t)$ for a.e. $(z, t)$, see Proposition 2.6 and Theorem 4.9 in Brezis [1]. Now we observe that

$$
\tilde{G}_{m}\left(z,\left|u_{m}(z, t)\right|\right)-\tilde{G}\left(z,\left|u_{m}(z, t)\right|\right)=\int_{0}^{\left|u_{m}\right|}\left(G_{m}(z, \sigma)-G(z, \sigma)\right) d \sigma \rightarrow 0
$$

pointwise as $m \rightarrow \infty$. Similarly $\tilde{G}\left(z,\left|u_{m}(z, t)\right|\right)-\tilde{G}(z,|u(z, t)|) \rightarrow 0$ pointwise as $m \rightarrow \infty$, therefore $\tilde{G_{m}}\left(z,\left|u_{m}(z, t)\right|\right)-\tilde{G}(z,|u(z, t)|) \rightarrow 0$ pointwise as $m \rightarrow$ $\infty$. By Fatou's Lemma, we have

$$
\int_{\mathbb{C}^{n}} \tilde{G}(z,|u(z, t)|) d z \leq \liminf \int_{\mathbb{C}^{n}} \tilde{G_{m}}\left(z,\left|u_{m}(z, t)\right|\right) d z .
$$

By above observations and energy conservation $E_{m}\left(u_{m}(\cdot, t)\right)=E_{m}(f)$, we have $E(u(\cdot, t)) \leq \liminf E_{m}\left(u_{m}(\cdot, t)\right)=\lim _{m \rightarrow \infty} E_{m}(f)=E(f)$ for $t \in\left(t_{0}-T, t_{0}+\right.$ $T)$. This shows that $t \rightarrow E(u(\cdot, t))$ has local maximum at $t_{0}$. But we can 
repeat this argument with any point in $\left(T_{*}, T^{*}\right)$, therefore $t \rightarrow E(u(\cdot, t))$ has local maximum at every point of $\left(T_{*}, T^{*}\right)$. Since $t \rightarrow E(u(\cdot, t))$ is continuous, therefore $E(u(\cdot, t))=E(f)$ for every $t \in\left(T_{*}, T^{*}\right)$.

Uniqueness: Uniqueness in $C\left(\left(T_{*}, T^{*}\right), \tilde{W}_{\mathcal{L}}^{1,2}\left(\mathbb{C}^{n}\right)\right) \cap L_{\text {loc }}^{\gamma}\left(\left(T_{*}, T^{*}\right), \tilde{W}_{\mathcal{L}}^{1, \rho}\left(\mathbb{C}^{n}\right)\right)$ will follow from estimate (2.7) with $m=0$ in Proposition 2.5, see uniqueness in [6].

Blowup alternative: We prove blowup alternative by method of contradiction. Let us assume that $T^{*}<\infty$ and $u \in L^{q}\left(\left(t_{0}, T^{*}\right), \tilde{W}_{\mathcal{L}}^{1, p}\right)$ for some admissible pair $(q, p)$ with $2<p$ and $\frac{1}{q}=n\left(\frac{1}{2}-\frac{1}{p}\right)$. Since $2<p<\frac{2 n}{n-1}$, $n \geq 2$, so $p<2 n$. We choose admissible pair $\left(q_{1}, p_{1}\right)$ as follows

$$
\frac{1}{p_{1}^{\prime}}=\frac{1}{p_{1}}+\frac{2}{n-1}\left(\frac{1}{p}-\frac{1}{2 n}\right), \frac{1}{q_{1}^{\prime}}=\frac{1}{q_{1}}+\frac{2}{n-1} \frac{1}{q} .
$$

Let us choose $s$ and $t$ such that $t_{0} \leq s<t<T^{*}$. Since $\left|S_{j} G(z, u(z, t))\right| \leq$ $C|u|^{\frac{2}{n-1}}\left(|u|+\left|Z_{j} u\right|+\left|\bar{Z}_{j} u\right|\right)$ for $S_{j}=I d, Z_{j}, \bar{Z}_{j}(1 \leq j \leq n$ ) (see Lemma 3.4 in [7]), therefore by Lemma 2.1 and Hölder's inequality we see that

$$
\|G(z, u(z, \tau))\|_{L^{q_{1}^{\prime}\left((s, t), \tilde{W}_{\mathcal{L}}^{1, p_{1}^{\prime}}\right)}} \leq C\|u\|_{L^{q_{1}}\left((s, t), \tilde{W}_{\mathcal{L}}^{1, p_{1}}\right)}\|u\|_{L^{q}\left((s, t), \tilde{W}_{\mathcal{L}}^{1, p}\right)}^{\frac{2}{n-1}} .
$$

Since $\left(t_{0}, T^{*}\right)$ is a bounded interval, so we can choose constant $C$ independent of $s$ and $t$, where $t_{0} \leq s<t<T^{*}$. Now we see that

$$
u(z, \tau)=e^{-i(\tau-s) \mathcal{L}} u(\cdot, s)(z)-i \int_{s}^{\tau} e^{-i\left(\tau-s_{1}\right) \mathcal{L}} G\left(z, s_{1}, u\left(z, s_{1}\right)\right) d s_{1} .
$$

Therefore we deduce from Strichartz estimates that

$$
\|u\|_{L^{q_{1}}\left((s, t), \tilde{W}_{\mathcal{L}}^{1, p_{1}}\right)} \leq C\|u(\cdot, s)\|_{\tilde{W}_{\mathcal{L}}^{1,2}}+C\|u\|_{L^{q_{1}}\left((s, t), \tilde{W}_{\mathcal{L}}^{1, p_{1}}\right)}\|u\|_{L^{q}\left((s, t), \tilde{W}_{\mathcal{L}}^{1, p}\right)}^{\frac{2}{n-1}} .
$$

where constant $C$ is independent of $s$ and $t$. Since $p \neq 2$, so $q<\infty$ and $u \in L^{q}\left(\left(t_{0}, T^{*}\right), \tilde{W}_{\mathcal{L}}^{1, p}\left(\mathbb{C}^{n}\right)\right)$, therefore we choose $s$ sufficiently close to $T^{*}$ such that

$$
C\|u\|_{L^{q}\left(\left(s, T^{*}\right), \tilde{W}_{\mathcal{L}}^{1, p}\left(\mathbb{C}^{n}\right)\right)}^{\frac{2}{n-1}} \leq \frac{1}{2}
$$

Therefore we get

$$
\|u\|_{L^{q_{1}}\left((s, t), \tilde{W}_{\mathcal{L}}^{1, p_{1}}\left(\mathbb{C}^{n}\right)\right)} \leq 2 C\|u(\cdot, s)\|_{\tilde{W}_{\mathcal{L}}^{1,2}} .
$$

Since RHS is independent of $t \in\left(s, T^{*}\right)$, so we have $u \in L^{q_{1}}\left(\left(s, T^{*}\right), \tilde{W}_{\mathcal{L}}^{1, p_{1}}\left(\mathbb{C}^{n}\right)\right)$. Therefore $u \in L^{q_{1}}\left(\left(t_{0}, T^{*}\right), \tilde{W}_{\mathcal{L}}^{1, p_{1}}\right)$ and $G(z, u(z, \tau)) \in L^{q_{1}^{\prime}}\left(\left(t_{0}, T^{*}\right), \tilde{W}_{\mathcal{L}}^{1, p_{1}^{\prime}}\right)$ follows from (3.15). Now from Strichartz estimates and (3.11) $), u \in L^{\tilde{q}}\left(\left(t_{0}, T^{*}\right)\right.$, 
$\left.\tilde{W}_{\mathcal{L}}^{1, \tilde{p}}\left(\mathbb{C}^{n}\right)\right) \cap C\left(\left[t_{0}, T^{*}\right], \tilde{W}_{\mathcal{L}}^{1,2}\left(\mathbb{C}^{n}\right)\right)$ for every admissible pair $(\tilde{q}, \tilde{p})$. Now by considering $T^{*}$ as a initial time and by local existence argument, we get contradiction to maximality of $T^{*}$.

Local stability: Let $f_{k} \rightarrow f$ in $\tilde{W}_{\mathcal{L}}^{1,2}\left(\mathbb{C}^{n}\right)$. Then for each $T>0$,

$$
\left\|e^{-i\left(t-t_{0}\right) \mathcal{L}}\left(f-f_{k}\right)\right\|_{L^{\gamma}\left(I_{T}, \tilde{W}_{\mathcal{L}}^{1, \rho}\left(\mathbb{C}^{n}\right)\right)} \leq C\left\|f-f_{k}\right\|_{\tilde{W}_{\mathcal{L}}^{1,2}\left(\mathbb{C}^{n}\right)} \rightarrow 0 \text { as } k \rightarrow \infty
$$

where $I_{T}=\left(t_{0}-T, t_{0}+T\right)$. Therefore for given $\delta>0$ in (3.5), choose $T(\delta)$ sufficiently small such that

$$
\left\|e^{-i\left(t-t_{0}\right) \mathcal{L}} f\right\|_{L^{\gamma}\left(I_{T}, \tilde{W}_{\mathcal{L}}^{1, \rho}\right)} \leq \frac{\delta}{2}
$$

and choose $k$ sufficiently large so that

$$
\left\|e^{-i\left(t-t_{0}\right) \mathcal{L}}\left(f-f_{k}\right)\right\|_{L^{\gamma}\left(I_{T}, \tilde{W}_{\mathcal{L}}^{1, \rho}\left(\mathbb{C}^{n}\right)\right)} \leq C\left\|f-f_{k}\right\|_{\tilde{W}_{\mathcal{L}}^{1,2}\left(\mathbb{C}^{n}\right)} \leq \frac{\delta}{2} .
$$

Therefore choose $k_{0}(T)$ so large such that

$$
\left\|e^{-i\left(t-t_{0}\right) \mathcal{L}} f_{k}\right\|_{L^{\gamma}\left(I_{T}, \tilde{W}_{\mathcal{L}}^{1, \rho}\left(\mathbb{C}^{n}\right)\right)} \leq \delta
$$

for $k \geq k_{0}(T)$.

Let $u$ and $\tilde{u_{k}}$ are solutions corresponding to initial values $f$ and $f_{k}$ at time $t_{0}$ respectively for $k \geq 1$. In view of estimates (3.9) and (3.10), $u, \tilde{u_{k}}$ will satisfy following estimates

$$
\begin{aligned}
\|u\|_{L^{\gamma}\left(I_{T}, \tilde{W}_{\mathcal{L}}^{1, \rho}\left(\mathbb{C}^{n}\right)\right)} & \leq 2 \delta \\
\|u\|_{L^{q}\left(\left(t_{0}, t_{0}+T\right), \tilde{W}_{\mathcal{L}}^{1, p}\left(\mathbb{C}^{n}\right)\right)} & <\infty \\
\sup _{k \geq k_{0}(T)}\left\|\tilde{u}_{k}\right\|_{L^{\gamma}\left(I_{T}, \tilde{W}_{\mathcal{L}}^{1, \rho}\left(\mathbb{C}^{n}\right)\right)} & \leq 2 \delta \\
\sup _{k \geq k_{0}(T)}\left\|\tilde{u}_{k}\right\|_{L^{q}\left(I_{T}, \tilde{W}_{\mathcal{L}}^{1, p}\left(\mathbb{C}^{n}\right)\right)} & <\infty
\end{aligned}
$$

where $(q, p)$ be any admissible pair. Now from Strichartz estimates and Lemma 2.2 ,

$$
\begin{aligned}
\left\|u-\tilde{u}_{k}\right\|_{L^{\gamma}\left(I_{T}, L^{\rho}\right)}= & \left\|\mathcal{H} u-\mathcal{H} \tilde{u}_{k}\right\|_{L^{\gamma}\left(I_{T}, L^{\rho}\right)} \\
\leq & C\left\|f-f_{k}\right\|_{\tilde{W}_{\mathcal{L}}^{1,2}\left(\mathbb{C}^{n}\right)}+C\left\|G(z, u)-G\left(z, \tilde{u_{k}}\right)\right\|_{L^{\gamma^{\prime}}\left(I_{T}, L^{\rho^{\prime}}\right)} \\
\leq & C\left\|f-f_{k}\right\|_{\tilde{W}_{\mathcal{L}}^{1,2}\left(\mathbb{C}^{n}\right)}+C\left\|u-\tilde{u_{k}}\right\|_{L^{\gamma}\left(I_{T}, L^{\rho}\left(\mathbb{C}^{n}\right)\right)} \times \\
& \left(\|u\|_{L^{\gamma}\left(I_{T}, \tilde{W}_{\mathcal{L}}^{1, \rho}\left(\mathbb{C}^{n}\right)\right)}+\left\|\tilde{u}_{k}\right\|_{L^{\gamma}\left(I_{T}, \tilde{W}_{\mathcal{L}}^{1, \rho}\left(\mathbb{C}^{n}\right)\right)}\right)^{\frac{2}{n-1}} .
\end{aligned}
$$

From (3.5) and (3.9),

$$
C\left(\|u\|_{L^{\gamma}\left(I_{T}, \tilde{W}_{\mathcal{L}}^{1, \rho}\left(\mathbb{C}^{n}\right)\right)}+\|{\tilde{u_{k}}}_{L^{\gamma}\left(I_{T}, \tilde{W}_{\mathcal{L}}^{1, \rho}\left(\mathbb{C}^{n}\right)\right)}\right)^{\frac{2}{n-1}} \leq \frac{1}{2} .
$$


Therefore $\left\|u-\tilde{u_{k}}\right\|_{L^{\gamma}\left(I_{T}, L^{\rho}\right)} \leq 2 C\left\|f-f_{k}\right\|_{\tilde{W}_{\mathcal{L}}^{1,2}\left(\mathbb{C}^{n}\right)} \rightarrow 0$ as $k \rightarrow \infty$. Since $\left\{\tilde{u}_{k}\right\}$ is a bounded sequence in $L^{\gamma}\left(I_{T}, \tilde{W}_{\mathcal{L}}^{1, \rho}\left(\mathbb{C}^{n}\right)\right)$, therefore from Lemma 2.2 with $m=0,\left\|G(z, u(z, t))-G\left(z, \tilde{u_{k}}(z, t)\right)\right\|_{L^{\gamma^{\prime}}\left(I_{T}, L^{\rho^{\prime}}\left(\mathbb{C}^{n}\right)\right)} \rightarrow 0$ as $j \rightarrow \infty$. Since $\mathcal{H} u=$ $u, \mathcal{H} \tilde{u}_{k}=\tilde{u}_{k}$, therefore from Strichartz estimates, $\left\|u-\tilde{u}_{k}\right\|_{L^{q}\left(I_{T}, L^{p}\left(\mathbb{C}^{n}\right)\right)} \rightarrow 0$ as $k \rightarrow \infty$ for every admissible pair $(q, p)$.

Note that $\left(\partial_{x_{j}}-\frac{i y_{j}}{2}\right)=\frac{1}{2}\left(Z_{j}-\bar{Z}_{j}\right)$ and $\left(\partial_{y_{j}}+\frac{i x_{j}}{2}\right)=\frac{i}{2}\left(Z_{j}+\bar{Z}_{j}\right)$. For $S=\left(\partial_{x_{j}}-\frac{i y_{j}}{2}\right),\left(\partial_{y_{j}}+\frac{i x_{j}}{2}\right)$ and using the notation $\psi_{(k)}=\psi\left(z,\left|\tilde{u}_{k}(z, t)\right|\right)$ (see (4.17) in [6]), we have

$$
\begin{aligned}
S\left(G_{(k)}-G\right)= & \psi_{(k)} S\left(\tilde{u}_{k}-u\right)+\left(\psi_{(k)}-\psi\right) S u+\left(\partial_{j} \psi_{(k)}\right)\left(\tilde{u}_{k}-u\right) \\
& +\left(\partial_{j} \psi_{(k)}-\partial_{j} \psi\right) u+\left(\partial_{2 n+1} \psi_{(k)}\right) \tilde{u}_{k} \Re\left(\frac{\bar{u}_{k}}{\left|\tilde{u}_{k}\right|} S\left(\tilde{u}_{k}-u\right)\right) \\
& +\left(\partial_{2 n+1} \psi_{(k)}\right) \tilde{u}_{k} \Re\left(\frac{\overline{\tilde{u}_{k}}}{\left|\tilde{u}_{k}\right|} S u\right)-\left(\partial_{2 n+1} \psi\right) u \Re\left(\frac{\bar{u}}{|u|} S u\right)
\end{aligned}
$$

where $\partial_{j}=\partial_{x_{j}}$ for $S=\left(\partial_{x_{j}}-\frac{i y_{j}}{2}\right)$ and $\partial_{j}=\partial_{y_{j}}$ for $S=\left(\partial_{y_{j}}+\frac{i x_{j}}{2}\right), 1 \leq j \leq n$.

Using the assumption (1.4) on $\psi$, Lemma 2.1, and by similar computations as used in Lemma 2.2 and Proposition 2.6, we have

$$
\begin{gathered}
\left\|\psi_{(k)} S\left(\tilde{u}_{k}-u\right)\right\|_{L^{\gamma^{\prime}}\left(I_{T}, L^{\rho^{\prime}}\right)} \leq C\left\|S\left(\tilde{u}_{k}-u\right)\right\|_{L^{\gamma}\left(I_{T}, L^{\rho}\right)}\left\|\tilde{u}_{k}\right\|_{L^{\gamma}\left(I_{T}, \tilde{W}_{\mathcal{L}}^{1, \rho}\right)}^{\frac{2}{n-1}} \\
\left\|\left(\partial_{j} \psi_{(k)}\right)\left(\tilde{u}_{k}-u\right)\right\|_{L^{\gamma^{\prime}}\left(I_{T}, L^{\rho^{\prime}}\right)} \leq C\left\|\tilde{u}_{k}-u\right\|_{L^{\gamma}\left(I_{T}, L^{\rho}\right)}\left\|\tilde{u}_{k}\right\|_{L^{\gamma}\left(I_{T}, \tilde{W}_{\mathcal{L}}^{1, \rho}\right)}^{\frac{2}{n-1}} \\
\left\|\left(\partial_{2 n+1} \psi_{(k)}\right) \tilde{u}_{k} \Re\left(\frac{\overline{\tilde{u}_{k}}}{\left|\tilde{u}_{k}\right|} S\left(\tilde{u}_{k}-u\right)\right)\right\|_{L^{\gamma^{\prime}}\left(I_{T}, L^{\rho^{\prime}}\right)} \\
\leq C\left\|S\left(\tilde{u}_{k}-u\right)\right\|_{L^{\gamma}\left(I_{T}, L^{\rho}\right)}\left\|\tilde{u}_{k}\right\|_{L^{\gamma}\left(I_{T}, \tilde{W}_{\mathcal{L}}^{1, \rho}\right)}^{\frac{2}{n-1}} .
\end{gathered}
$$

Since $\left\|\tilde{u}_{k}-u\right\|_{L^{\gamma}\left(I_{T}, L^{\rho}\left(\mathbb{C}^{n}\right)\right)} \rightarrow 0$ and $\left\{\tilde{u}_{k}\right\}$ is a bounded sequence in $L^{\gamma}\left(I_{T}, \tilde{W}_{\mathcal{L}}^{1, \rho}\right)$, therefore by second inequality in the above estimates, $\left(\partial_{j} \psi_{(k)}\right)\left(\tilde{u}_{k}-u\right) \rightarrow 0$ as $k \rightarrow \infty$ in $L^{\gamma^{\prime}}\left(I_{T}, L^{\rho^{\prime}}\left(\mathbb{C}^{n}\right)\right)$. Since $G$ is $C^{1}$, so in view of the condition (1.4) on $\psi$ and Proposition 2.6, the sequences $\left(\psi_{(k)}-\psi\right) S u,,\left(\partial_{j} \psi_{(k)}-\partial_{j} \psi\right) u$ and $\left(\partial_{2 n+1} \psi_{(k)}\right) \tilde{u}_{k} \Re\left(\frac{\bar{u}_{k}}{\left|\tilde{u}_{k}\right|} S u\right)-\left(\partial_{2 n+1} \psi\right) u \Re\left(\frac{\bar{u}}{|u|} S u\right)$ converges to zero in $L^{\gamma^{\prime}}\left(I_{\tau}, L^{\rho^{\prime}}\right)$ as $k \rightarrow \infty$. Using these observations in (3.22), we get

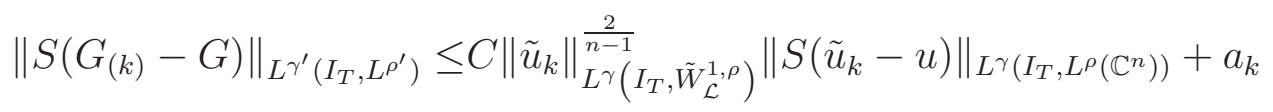

where $S=\left(\partial_{x_{j}}-\frac{i y_{j}}{2}\right),\left(\partial_{y_{j}}+\frac{i x_{j}}{2}\right)(1 \leq j \leq n)$ and $a_{k} \rightarrow 0$ as $k \rightarrow \infty$. Since $\left(\partial_{x_{j}}-\frac{i y_{j}}{2}\right)=\frac{1}{2}\left(Z_{j}-\bar{Z}_{j}\right)$ and $\left(\partial_{y_{j}}+\frac{i x_{j}}{2}\right)=\frac{i}{2}\left(Z_{j}+\bar{Z}_{j}\right)$, therefore we have

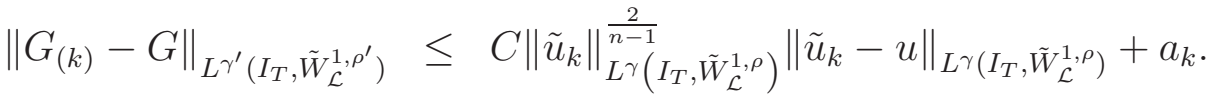


Now from Strichartz estimates and above estimate, we have

$\left\|\tilde{u}_{k}-u\right\|_{L^{\gamma}\left(I_{T}, \tilde{W}_{\mathcal{L}}^{1, \rho}\right)} \leq C\left\|f_{k}-f\right\|_{\tilde{W}_{\mathcal{L}}^{1,2}}+C\left\|\tilde{u}_{k}\right\|_{L^{\gamma}\left(I_{T}, \tilde{W}_{\mathcal{L}}^{1, \rho}\right)}^{\frac{2}{n-1}}\left\|\tilde{u}_{k}-u\right\|_{L^{\gamma}\left(I_{T}, \tilde{W}_{\mathcal{L}}^{1, \rho}\right)}+a_{k}$.

Now we choose $\delta>0$ sufficiently small such that it satisfies condition (3.5) and

$$
C(2 \delta)^{\frac{2}{n-1}} \leq \frac{1}{2}
$$

where constant $C$ is appearing in the inequality (3.24). Note that $T$ depends on $\delta$ through (3.16). Therefore from estimates (3.20) and (3.24), we have

$$
\left\|\tilde{u}_{k}-u\right\|_{L^{\gamma}\left(I_{T}, \tilde{W}_{\mathcal{L}}^{1, \rho}\right)} \leq 2 C\left\|f_{k}-f\right\|_{\tilde{W}_{\mathcal{L}}^{1,2}}+2 a_{k} \rightarrow 0
$$

as $k \rightarrow \infty$. Now from estimates (3.23), (3.18) and (3.20)

$$
\left\|G_{(k)}-G\right\|_{L^{\gamma^{\prime}}\left(I_{T}, \tilde{W}_{\mathcal{L}}^{1, \rho^{\prime}}\right)} \rightarrow 0
$$

as $k \rightarrow \infty$. From Strichartz estimates, $\left\|\tilde{u}_{k}-u\right\|_{L^{q}\left(I_{T}, \tilde{W}_{\mathcal{L}}^{1, p}\right)}=\left\|\mathcal{H} \tilde{u}_{k}-\mathcal{H} u\right\|_{L^{q}\left(I_{T}, \tilde{W}_{\mathcal{L}}^{1, p}\right)}$ $\rightarrow 0$ as $k \rightarrow \infty$ for every admissible pair $(q, p)$.

Stability: Let $\left(T_{*, k}, T_{k}^{*}\right)$ be the maximal interval for the solutions $\tilde{u}_{k}$ and $I \subset\left(T_{*}, T^{*}\right)$ be a compact interval. The key idea is to extend the local stability result proved above to the interval $I$ by covering it with finitely many intervals obtained by successive application of the above local stability argument. This is possible provided $\tilde{u}_{k}$ is defined on $I$, for all but finitely many $k$. In fact, we prove $I \subset\left(T_{*, k}, T_{k}^{*}\right)$ for all but finitely many $k$.

Without loss of generality, we assume that $t_{0} \in I=[a, b]$, and give a proof by the method of contradiction. Suppose there exist infinitely many $T_{k_{m}}^{*} \leq b$ and let $c=\liminf T_{k_{m}}^{*}$. Then for $\epsilon>0,\left[t_{0}, c-\epsilon\right] \subset\left[t_{0}, T_{k_{m}}^{*}\right)$ for all $k_{m}$ sufficiently large and $\tilde{u}_{k_{m}}$ are defined on $\left[t_{0}, c-\epsilon\right]$.

By compactness, the local stability result proved above can be extended to the interval $\left[t_{0}, c-\epsilon\right]$.

For given $\delta>0$, choose $\epsilon>0$ sufficiently small such that

$$
\begin{array}{r}
\left\|e^{-i(t-(c-\epsilon)) \mathcal{L}} u(\cdot, c-\epsilon)-e^{-i(t-(c-\epsilon)) \mathcal{L}} u(\cdot, c)\right\|_{L^{\gamma}\left((c-\epsilon, c+\epsilon), \tilde{W}_{\mathcal{L}}^{1, \rho}\right)} \\
\leq C\|u(\cdot, c-\epsilon)-u(\cdot, c)\|_{\tilde{W}_{\mathcal{L}}^{1,2}} \leq \frac{\delta}{6} \\
\left\|e^{-i(t-(c-\epsilon)) \mathcal{L}} u(\cdot, c)-e^{-i(t-c) \mathcal{L}} u(\cdot, c)\right\|_{L^{\gamma}\left((c-\epsilon, c+\epsilon), \tilde{W}_{\mathcal{L}}^{1, \rho}\right)} \\
\leq C\left\|e^{-i \epsilon t \mathcal{L}} u(\cdot, c)-u(\cdot, c)\right\|_{\tilde{W}_{\mathcal{L}}^{1,2}} \leq \frac{\delta}{6} \\
\left\|e^{-i(t-c) \mathcal{L}} u(\cdot, c)\right\|_{L^{\gamma}\left((c-\epsilon, c+\epsilon), \tilde{W}_{\mathcal{L}}^{1, \rho}\right)} \leq \frac{\delta}{6} .
\end{array}
$$


Now we choose $k_{0}(\epsilon)$ such that following estimate holds for all $k \geq k_{0}$

$$
\begin{gathered}
\left\|e^{-i(t-(c-\epsilon))} \tilde{u}_{k_{m}}(\cdot, c-\epsilon)-e^{-i(t-(c-\epsilon))} u(\cdot, c-\epsilon)\right\|_{L^{\gamma}\left((c-\epsilon, c+\epsilon), \tilde{W}_{\mathcal{L}}^{1, \rho}\right)} \\
\leq C\left\|\tilde{u}_{k_{m}}(\cdot, c-\epsilon)-u(\cdot, c-\epsilon)\right\|_{\tilde{W}_{\mathcal{L}}^{1,2}} \leq \frac{\delta}{2} .
\end{gathered}
$$

Therefore $\left\|e^{-i(t-(c-\epsilon))} \tilde{u}_{k_{m}}(\cdot, c-\epsilon)\right\|_{L^{\gamma}\left((c-\epsilon, c+\epsilon), \tilde{W}_{\mathcal{L}}^{1, \rho}\right)} \leq \delta$ for all $k_{m} \geq k_{0}$. Now by local existence argument (see (3.6) $), \tilde{u}_{k_{m}}$ is defined on $\left(t_{0}, c+\epsilon\right)$ and therefore $T_{k_{m}}^{*} \geq c+\epsilon$ for all $k_{m} \geq k_{0}$, hence contradicts the fact that $\lim \inf T_{k_{m}}^{*}=c$.

Similarly we can show that $\left[a, t_{0}\right] \subset\left(T_{*, k}, t_{0}\right]$ for all but finitely many $k$ which completes the proof of stability.

Acknowledgements: Author wishes to thank the Harish-Chandra Research institute, the Dept. of Atomic Energy, Govt. of India, for providing excellent research facility.

\section{REFERENCES}

[1] H. Brezis, Functional Analysis, Sobolev Spaces and Partial Differential Equations, Universitext Springer, New York, 2011.

[2] T. Cazennave, Semilinear Schrödinger Equations, Courant Lecture Notes in Mathematics, 2003.

[3] T. Cazenave, F.B. Weissler, Some remarks on the nonlinear Schrdinger equation in the critical case, Nonlinear Semigroups, Partial Differential Equations, and Attractors, .L. Gill and W.W. Zachary (eds.), Lecture Notes in Math. 1394, Springer, New York, 1989, 18-29.

[4] J. Ginibre, G. Velo, On a Class of Nonlinear Schrödinger Equations. I. The Cauchy Problem, General Case, J. Funt. Anal, Vol 32, (1979),1-32.

[5] P. K. Ratnakumar, On Schrodinger propagator for the special Hermite operator, $J$. Fourier Anal. Appl., Vol 14, (2008), 286-300.

[6] P. K. Ratnakumar, V. K. Sohani, Nonlinear Schrödinger equation for the twisted Laplacian, J. Funct. Anal. 265 (1) (2013) 1-27.

[7] P. K. Ratnakumar, V. K. Sohani, Nonlinear Schrödinger equation for the twisted Laplacian-global well posedness preprint.

[8] R. S. Strichartz, Harmonic analysis as spectral theory of Laplacians, J. Funct. Anal., Vol. 87, (1989) 51-148.

[9] S. Thangavelu, Lectures on Hermite and Laguerre expansions, Mathematical notes, 42, Princeton Univ. press, Princeton.(1993).

[10] S. Thangavelu, Harmonic Analysis on the Heisenberg Group, Progress in Math. Vol. 154, Birkhauser (1998).

[11] T. Tao, Nonlinear dispersive equations. Local and global analysis. AMS, CBMS Regional Conference Series in Math., 106, (2006).

[12] Z. Zhang, S. Zheng, Strichartz estimates and local wellposedness for the Schrödinger equation with the twisted sub-Laplacian, Proc. Centre Math. Appl. Austral. Nat. Univ., Volume 44, 2010.

Harish-Chandra Research Institute, Allahabad-211019 India

E-mail address: sohani@hri.res.in 